\title{
Multi-Agent Simulations of Intra-colony Violence in Ants
}

\author{
Kit Martin $^{1}$ and Pratim Sengupta ${ }^{2 \star}$ \\ 1 Northwestern University, Evanston IL 60202, USA \\ 2 Mind, Matter \& Media Lab, University of Calgary, Calgary AB T2N 1N4, Canada \\ kitmartin@u.northwestern.edu, pratim.sengupta@ucalgary.ca
}

\begin{abstract}
This paper seeks to elucidate key aspects of a rarely-studied interaction in ant colonies - intra-colony violence - using multi-agentbased computational simulations. A central finding is that intra-colony violence is heritable, though not prevalent. Results from our simulations reveal specific conditions in which such infrequent forms of violence occur and can be inherited, which in turn helps us understand why Atta cephalotes may persist killing colony members, even though it dampens colony carrying capacity. We also discuss the concerns and implications of our work for modeling conflict and violence more broadly, which in turn raises questions about the ontological nature of the computational and evolutionary models.
\end{abstract}

Keywords: Multi-Agent Simulations, Emergence, Ant Colonies, Violence.

\section{Introduction}

Intra-specific violence is a common phenomenon among ant colonies, as reported by biologists such as Whitehouse's [41] work on Atta laevigata. Nearly all observations of violence in ants are observations of violence among colonies within the same species [26] rather than within a colony, because generally, within ant colonies, sterile workers cooperate to rear colonial young [45]. Instances of intra-colony violence are present in Atta cephalotes colonies, as observed during fieldwork conducted by the first author (see figure 1). The goal of this paper is to present a set of computational experiments that shed light on the evolutionary stability of this significantly less studied form of violence within Atta cephalotes colonies. Nest-mates (i.e., members of the same colony) killing each other is an interesting case, because theoretically the benefits of cooperation should outweigh the benefits of intra-colony competition because of the associated net loss of sterile foraging members [37].

We investigate the following research question: How can intra-colony violence persist over generations in ant colonies in which all members are related,

\footnotetext{
* This research was supported by the US National Science Foundation under a CAREER Grant awarded to Pratim Sengupta (NSF CAREER 1150230). All opinions are the authors' and not endorsed by any organization.
} 


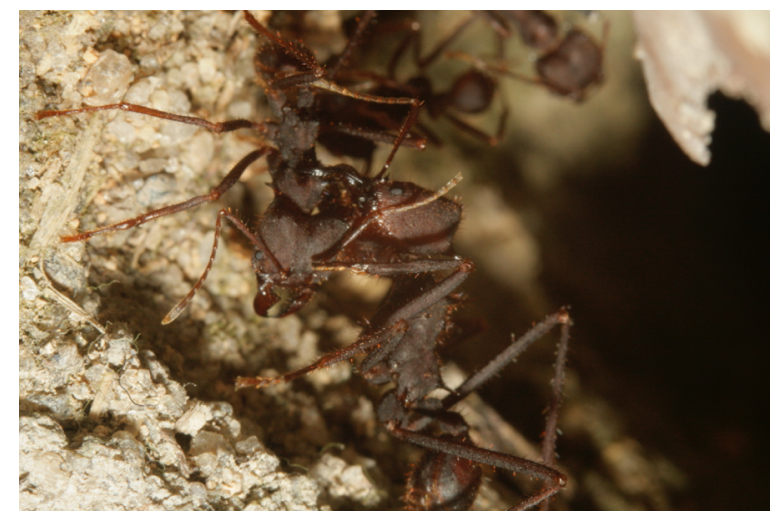

Fig. 1. Photo of two ants (Atta cephalotes) engaged in intra-colony conflict. Image credit: Kit Martin

and colony success is predicated on mutual cooperation and almost ubiquitous sterility? To answer this question, we developed a multi-agent-based simulation in NetLogo [42]. We conducted several computational experiments to help us identify circumstances where pure cooperation among nest-mates, in the face of aggressively violent behavior among nest-mates, sometimes loses from an evolutionary perspective. It is the identification of these specific circumstances, we posit, that stand as the main contribution of this paper. An implication of our work is the possibility of a deeper understanding of underlying mechanisms of cooperation and conflict (more broadly), with obvious cautions against misinterpretation and over-generalization, as we explain later.

Our open source NetLogo model is available via Open ABM [24], where following Grimm et al. [8], we have also attached a complete Overview, Design, Concepts, Details protocol to make modeling design decisions explicit, and thus more reproducible. In this paper, we first situate our work at the nexus of previous scholarship on ant biology and computational complexity. We then describe the basic version of the NetLogo model we began with, and how we modified this model to simulate three different scenarios that allowed us to investigate follow-up research questions. We conclude with some implications of our work for future research on modeling violence as an emergent system, grounded in an essential caution against unwarranted generalization.

\section{Background: Ant Biology}

\section{Individual Life Histories of Atta cephalotes}

Ants are a worldwide species that dominate many terrestrial ecosystems [14]. Ant colonies are composed of mostly sterile female workers, who protect the colony, collect food and take care of eggs in a cooperative structure, building a unit of selection larger than the individual $[15,45]$. There are over 15,680 distinct 
species of ants, with almost as many distinct patterns of life histories [6]. In this model, we modeled Atta cephalotes, the type of fungus growing ants the first author observed in field work. They collect leaves and flowers to feed to fungus farms within their subterranean nests [16]

In Atta cephalotes reproduction is handled either by an individual female ant or a small number of female ants, termed queens, who usually mate once and live for decades [28]. Atta cephalotes, like many other ants, have a communication system predominantly based on time-diminishing pheromones, but also including drumming, touching, and vision [18], and share their knowledge using tandem running [7]. While only using $10 \%$ of the colony for food foraging, colonies can provide the nutritional needs of the entire colony, including immobile larvae, or queens [5]. Ants' success, like other social animals, hinges on their ability to take advantage of ephemeral natural resources to rear young and pass on their genetic heritage to closely related offspring in neighboring colonies. The model used this life history information to inform the model creation as outlined in its ODD [24].

\section{Distinguishing "Friend" From "Foe" in Ant Colonies}

Ant colonies, like several other insect societies (e.g., honeybees), are seen as examples of superorganisms $[15,31,40]$. Originally coined by Morton Wheeler [40] to denote insect societies that "possess features of organization analogous to the physiological processes of individual organisms" [31, p. 548], it indicates societies of organisms such as ants and honeybees that form a cooperative unit to propagate their genes. As Seeley noted, the analogy here is the classification of a group of cells as an organism when the cells form a cooperative unit to propagate their genes [31]. But this also suggests that most insect societies are not perfect superorganisms because there is usually intense intragroup conflict when members compete for reproductive success [38].

That being said, superorganisms have also been defined as insect societies that have very low aggression towards nestmates but high aggression towards outsiders [35]. As Moffett said "All societies are characterized by the capacity of their members to distinguish one another from outsiders" [27, p. 1]. In ant colonies, members recognize each other through odor cues contained in hydrocarbons, or oils, on the ant's exoskeleton [3, 15, 19, 22, 25, 39, 47, 48]. They also employ the hydrocarbons as home range marking [21], which allows colony mates to distinguish each other without having to actually have met before [27]. This "anonymous" scent based recognition in ants has two effects. First, it means larger groups of ants can work together altruistically on an effort and form a society without intra-colony violence or individual recognition. Second, it means errors may exist in recognizing colony or colony-mates, as the results have shown that in turn may lead to intra-colonial violence [39]. 


\section{Intra-Colony Violence}

Examples of violence between nest mates have been documented in a few different ant species [30]. Linepithema humile, Argentine ants, have been induced to kill colony mates, possibly through a change in diet [23]. In our field observations, we observed nestmates of $A$. cephalotes kill each other near Manaus, Brazil. Kin selection, competition among the minutely diverse genetic makeups of ants in a colony, provides a rationale for the persistence of intra-colony competition. Kin selection takes a variety of forms, including queens in the same colony that may kill each other for the right to control the genetic heritage of offspring, mostly sterile workers that may compete for the rights to lay male eggs [4], or "varieties of strategies that even nonlaying workers might employ to ensure genetic success" [30, p. 171], such as killing or selectively starving less related eggs. In short, while on the one hand, the super-organism attacking itself seems intuitively like a negative externality that should result in negative colonial fitness, that would be selected against. On the other hand, selfishness may still have benefits in an altruistic ant colony [30]. Of particular interest to us is the question whether such "cross-purposes" (p. 171) are merely glitches in an otherwise functional and highly adaptive super-organism structure, or themselves have utility evolutionarily speaking.

\section{Theoretical Framework}

\section{High-Leverage Points in Complex Systems}

Holland [13] argued that an ant nest is an apt example of "emergence of complex large-scale behaviors from the aggregate interactions of less complex agents" (p. 11). Such emergent systems can be understood as systems with high leveragepoints, where a local or an individual-level interaction can have systemic impact [12]. In the game of chess, which is also an example of a complex adaptive system, one such leverage point is a gambit [12], where a player sacrifices a pawn early in the game to set up the board for improved late game position. In an immune system, an example is a vaccine [13], where immunity to disease in the whole body results from prior exposure to microscopic pathogens. In both examples, "a small input can produce major predictable, directed change - an amplifier effect- -.." [13, p. 5]. When discovered, these high leverage points cause state-changes, i.e., the macro-scale environment changes appreciably due to small changes at the individual-level. In war, while there are points of leverage, they are difficult to identify. Solvit described war as a complex adaptive system but one without methodically delineated levers of intervention [33]. We posit that violence in ant colonies also reflects this feature of complex systems. A contribution of this paper is that we propose how varying killing rates in the colony can help us identify such a high leverage point, as it can lead to an evolutionarily significant, aggregate-level change for the colony. 


\section{Inclusive Fitness and Natural Selection}

In sociobology, the classic equation that models inclusive fitness is often used to measure fitness in social animals $[9,10]$ is shown below:

$$
r b>c
$$

This equation is known as Hamilton's rule. It helps us understand how altruism evolves or can be maintained in a selfish world. In this equation, $r$ represents the relatedness between the social partners, and can also be understood as a genotype or a collection of genetic material on a DNA strand that is passed to the subsequent generation. Hamilton's rule represents the idea that the benefits to the recipients of an altruistic act (b), weighted by the relatedness between the social partners (r), must exceed the costs to the altruists c, in order for altruistic genotypes to be passed on or inherited over by future generations over nonaltruistic ones.

Researchers have shown that this equation holds true for observations of inter-colony violence $[26,41]$. That is, one would expect two or more colonies competing with each other to fight over sources of food, and thus genes that cause functions leading to reproductive success to persist. However, the question at hand is as follows: how could intra-colony violence - i.e., members of the same colony killing each other - be evolutionarily beneficial for the colony? Is it even possible that the existence of colony members who practice the "kill my nestmate" behavior, in some circumstance, has a "benefit" to the colony? The equation for us, in such cases, would be as follows:

$$
r(k) b>c
$$

Here $r(k)$ represents the rate at which killers are born in a given colony. How can such a scenario be understood from the perspective of inclusive fitness and natural selection? What implications may such evolutionary occurrences have for our broader understanding of modeling violence as an emergent system as well as using evolutionary approaches? These are the questions that we are investigating in this paper.

\section{A Necessary Caution Against Over-generalization}

The notion of inclusive fitness is a well-accepted model for studying altruism in social insects $[11,44]$ and even human societies [36]. Altruism is defined as the benefit to others at a cost to the individual. In agent-based computational models of evolutionary biology, this is usually represented in the form of the transfer of genetic material at the cost of physical demise of the individual agent. Given this, for our present work, perhaps the most bizarre and dangerous question from a moral standpoint can be stated as follows: Could we view intra-colony violence as a form of altruism? That is, from a purely utilitarian perspective, could the emergent effect of ants killing nest mates be understood in light of a long-term successes for the colony as expressed through its inclusive genetic fitness? 
The unequivocal answer to this question is that the logic of cost-benefit analysis that underlies Hamilton's rule is a vastly reductive approach for modeling violence or mass-scale devastation. As moral philosophers point out, underlying the notion of "altruism" are axiomatic assumptions of marginal value and utilitarianism [34], requiring us to take the "point of view of the universe" rather than the perspective of the individual ethics [20]. By illustrating conditions in which infrequent mass violence may be inherited, our goal is not to justify such occurrences - on the contrary, it is to alert us to conditions under which violent acts - carried out by individuals - might come to hold marginal value over co-operation at the aggregate level. The ability to reveal such counter-intuitive connections between the individual and the aggregate levels of behavior in a complex emergent system is a particular affordance of multi-agent models [32, 43].

But at the same time, from an ontological perspective, we see this as a crisis rather than an affordance: positioning large-scale violence as a form of altruism only reveals a form of logical fallacy that has indeed proven to be dangerous in human history, as evident in the history of justifications of human genocides [17]. We discuss these ontological challenges and implications for modeling violence in human societies in more detail in the final section of the paper.

\section{The Basic Model}

The basic model consists of two agent-types ("breeds" in NetLogo terminology): red ants (Rants) and blue ants (Bants). At the beginning of the simulation, each ant starts with a built-in dietary preference, represented by a randomly selected number between 0 and 140 (using the HSB color scale). When an ant finds a plant that corresponds to her preference she picks it up (RedFood or BlueFood), gains a specific amount of energy (250 units), and returns a portion of that food to the home-nest for consumption by colony-mates (return-to-nest).

Food collection serves both the individual and the colony's survival. When the collective food in a colony reaches a threshold value, queen hatches one new baby ant. During a single time-step the colony can only gain upto one new ant from reproduction.

At the beginning of each simulation run, every colony has a specific proportion of killer ants represented by the variable $k$. In any encounter with other ants, each killer ant has a probability of $50 \%$ of killing the other ant. Other individual-level ant variables include age, their current $\mathrm{x}$ and $\mathrm{y}$ coordinates, and a Boolean variable representing whether they are looking for food or returning to nest.

Learning occurs in our model as follows: Ants share food preferences with other ants they encounter. Because the surrounding environment in the model changes every time step (see ODD), the food preferences for each ant also needs to update. While ants can randomly discover food, they also share their preferences if they have successfully found food. Social learning is therefore a mechanism for ants to dynamically alter their food preferences by learning from others' successes in foraging. This is represented by the function tandem-running [7], in 
which ants share their preferred food color with other ants if they are returning to the nest with food.

\section{The Computational Experiments: Three Scenarios}

As stated earlier, our overall research question is: How can violence and discord, when nest mates kill nest mates, persist in a social situation over generations in which all members are related and colony success is predicated on mutual cooperation and almost ubiquitous sterility? In order to answer this question, we modeled three scenarios by modifying the aforementioned "base" model. In Scenario 1, we modeled two competing colonies and in each colony, the queen only reproduced sterile workers. So in each colony, when the queen reproduced, she did not give birth to reproductively active males or females that could create new colonies. Thus, while the colony population grew over time, new colonies were not founded - i.e., there was no reproduction of the super-organism. The first scenario thus sought to investigate the comparative survival rates of colonies with and without killer ants, as we varied $k$. We refer to colonies without killer ants as non-violent or altruist colonies, represented visually as blue ants, while colonies with killer ants were represented as red ants. In Scenario 2, we initialized the model with five colonies that could reproduce both sterile workers and reproductively active males and females, who, in turn could also establish their own colonies. This allowed for super-organism reproduction. Experiments in this scenario sought to investigate how the heritability of $k$, as a result of reproduction both within and between colonies, effected the prevalence of both violent and non-violent ants. That is, in Scenario 2, we did not vary $k$ in each run, and instead observed how super-organism reproduction, along with other emergent factors (e.g., availability of food resources) effected the the value of $k$ over subsequent generations. Finally, experiments in Scenario 3 sought to investigate the role of learning by implementing a calamity in terms of sudden, drastic reduction of food resources in the environment while $k$ was not heritable but was varied systematically across simulation runs, as in Scenario 1.

The first scenario was run 165 times for 16,000 steps. The second scenario was run ten times for 43,810 steps. The Third scenario was run sixteen times for 16,000 steps.

\section{Results}

\section{Scenario 1: Intra-Colony Violence Without Super-Organism Reproduction}

An important finding of this set of experiments is that although on average the altruist colony can maintain a larger population, highly aggressive behavior can - albeit very infrequently - result in the total annihilation of competing colonies. This can be understood as follows. On an average, the non-violent colonies have a higher population than the murderous colonies. But in 6 out of 
165 trials, i.e., $3.6 \%$ of the time, the non-violent blue colony collapsed, while in the same trials - regardless of the value of $k$ - the red colony never collapsed. We also found that when the trials were run with two competing but non-violent colonies, neither colony ever collapsed, indicating that colony collapse cannot simply be attributed to resource exhaustion linked to competition from other colonies.

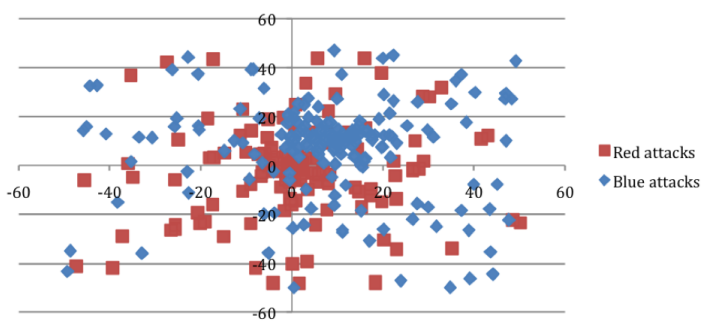

Fig. 2. Density-dependence of attacks: Attacks by Red and Blue killer ants are most prevalent at the colonies. Red colony is centered at $(0,0)$ and Blue colony is centered at $(10,10)$.

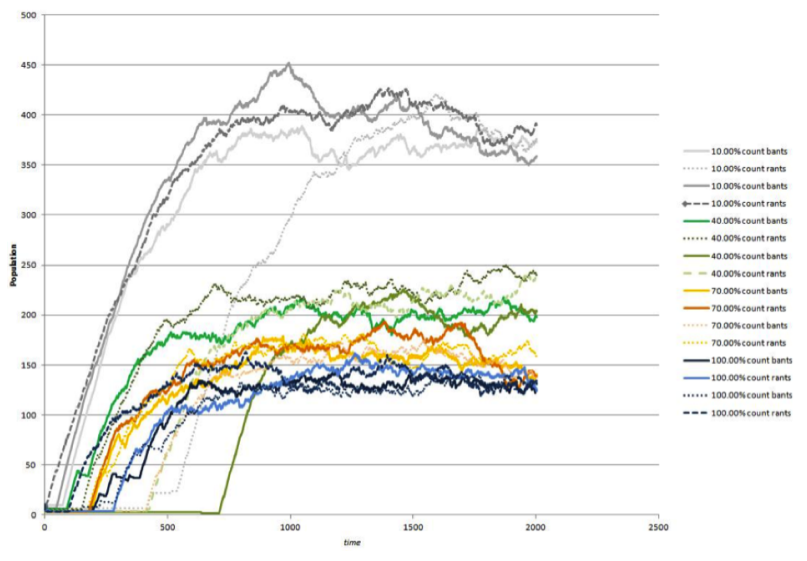

Fig. 3. The maximum population of ant colonies decreased as $k$ increased.

Another finding is that both attacks and social learning of food preferences are dependent on the density (i.e., spatial distribution) of agents, being most prevalent near the colony centers (Figures 2). Consequently, the nestgate regions (i.e., areas immediately surrounding the center of the colony), which are the most effective locations for sharing food preferences due to the highest concentrations of contacts between ants, are also the places where most lethal encounters hap- 
pen. Note that increasing the value of $k$ also reduces learning rates, which makes a colony less adaptive to its environment. In simulations with food scarcity the effect is greater, as adaptation to a changing environment is more crucial than in a stable state, and we investigate this in more detail in Scenario 3.

Finally, it is important to note that aggressive behavior also dampened population growth for the red colony. As shown in figure $3, k$ is increased from $10 \%$ to $100 \%$ in increments of $30 \%$, which has a cost for the red colony: higher values of $k$ are associated with with a lower maximum red ant population. For example, for $k=0$, the max population in the red colony was 3,000, while for $k=90 \%$, the max population was 120 .

\section{Scenario 2: Intra-Colony Violence with Super-Organism Reproduction}

For this scenario, we chose $k=20 \%$ as the starting value. This was based on sensitivity analysis which revealed that it is at and around this value, a violent colony can outperform non-violent colonies in terms of survival. When colonies were allowed to reproduce, we found that even though $20 \%$ of starting nests produced killers at the beginning of the simulation, by the end, only 58 of 1,167 (5\%) of nests produced killers $k=33 \%$, i.e., their queens birthing killer ants one third of the time. Comparison of average value of $k$ across all colonies at the beginning and the end of the experiment (43800 runs) reveals that it dropped from $40 \%$ to $2 \%$, while the average value of $k$ for violent colonies dropped from $67 \%$ to $33 \%$.

This indicates once such violent traits become heritable, they are difficult to be exterminated from the gene pool, even though they might only dominate the competition infrequently or rarely. One possible explanation is that when $k$ is heritable, even a single killing in a colony increases the likelihood of more killings both in the same colony and other colonies.

\section{Scenario 3: A Deeper Investigation of the Role of Resource Availability}

In this scenario, we worked with a version of the model used in Scenario 1, where $k$ was not heritable (set to 20\%) and involved two colonies (red and blue ants). We also introduced a food calamity event: we set red and blue food to 0 at time $t=10000$ steps. We chose the 10000 steps as an appropriate time for setting the calamity event based on sensitivity analysis that reveled that populations stabilize around this time. As shown in figure 4, after the food calamity, the effect of $k$ becomes clearer: $50 \%$ of red colonies and $37.5 \%$ of blue colonies collapsed after the food storage calamity.

We also found that it was around $k=20 \%$ that the effect of the calamity became pronounced in our simulation. This can be seen by comparing, for example, the populations of the two competing colonies at and around $\mathrm{t}=10000$ in figures 5 and 6 . These graphs show that at $k=20 \%$, the average population of red (violent) colony increases after the calamity at the expense of the average 


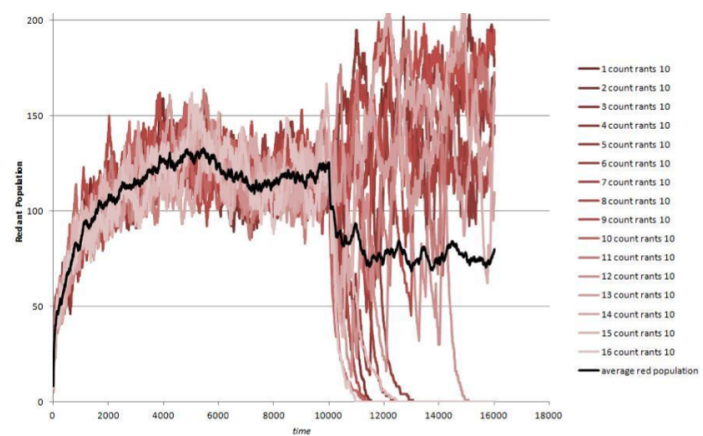

Fig. 4. Scenario 3: Average populations from 16 simulation runs for $k=20 \%$. Red colonies increase rapidly at first. Over time, growth plateaus and then drops to zero just as food resources deplete. Colonies must adjust quickly, or collapse. $50 \%(\mathrm{n}=8)$ colonies survive and their population explodes when the competing colonies die.

population of the blue (non-violent) colony. In contrast, for values less that $k=$ $20 \%$, we found no discernable difference in the average populations, an example of which is shown in figure 5 .

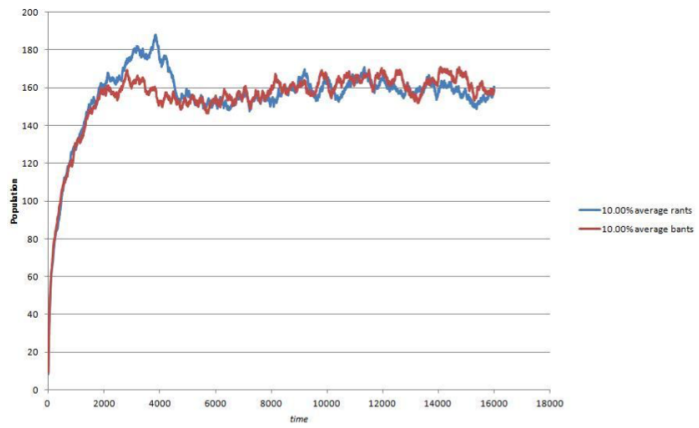

Fig. 5. Scenario 3: Average populations from 16 simulation runs at $k=10 \%$.

\section{Summary and Discussion}

\section{Summary of Results}

The results from our experiments can be summarized as follows:

- An important finding from the experiments in Scenario 1 is that although on average non-violent colonies can maintain a higher population, highly aggressive behavior in violent colonies can - albeit very infrequently result in the total annihilation of competing non-violent colonies. 


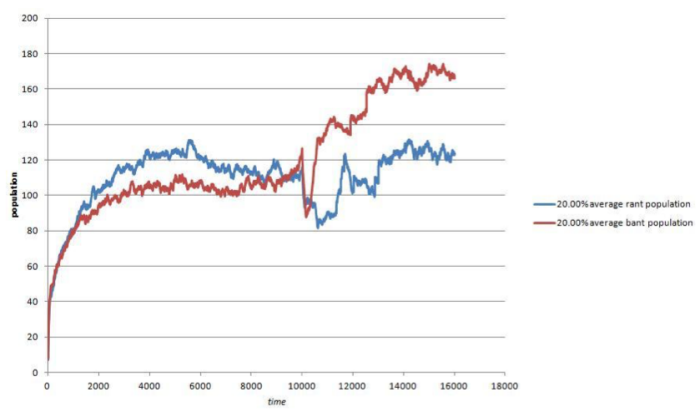

Fig. 6. Scenario 3: Average populations from 16 simulation runs at $k=20 \%$.

- Experiments in Scenario 1 also showed that the population in violent colonies never collapsed, while the population of non-violent colonies did collapse $3.6 \%$ of the time. However, aggressive behavior also lowers the population growth for violent colonies.

- Attacks between ants are density-dependent. Experiments in Scenario 1 showed that attacks by killer ants are most prevalent at and around the colony centers, and particularly at the nestgate regions, where the likelihood of ants sharing their food preferences is also high. Coupled with a low ability to distinguish between friend and foe, the presence of a higher number of ants in a particular location leads to higher number of attacks.

- Experiments in Scenario 2 showed that once violent traits become heritable, they are difficult to be exterminated from the gene pool, even though they might only dominate the competition infrequently or rarely.

- Experiments in Scenario 3 showed that effects of drastic reduction in availability of food results in differentiated effects on violent and non-violent colonies only for certain values of $k$ (around $k=20 \%$ ). For values significantly less than $20 \%$, the effect of a famine was nearly identical on both violent and non-violent colonies.

\section{Concerns and Implications for Modeling Human Societies}

It may be tempting to appropriate this work for modeling cooperation and violence in human societies. It is indeed true that multi-agent simulations can offer productive approaches for modeling complex human societies and experiences that may be otherwise difficult to model or understand. For example, multi-agent simulations can help us understand how gender and sexuality based marginalization and resilience are emergent, multi-level phenomena rather than individual-level experiences [29], and how apparently democratic systems of elections may be resting on deeply oligarchic foundations [46]. But, this kind of work may require a fundamentally more synthetic approach that integrates qualitative and subjective forms of human experiences with computational methods. This includes integrating narratives with data objects and algorithms for simulating complexity as integral parts of the computational model and modeling 
experience [29], and validating computational and algorithmic approaches with empirical work [46].

In absence of such synthetic methods, our current paper should not be interpreted as a model of human interactions. It is not an endorsement of the virtues of infrequent, mass violence. On the contrary, our work should serve as a reminder that even apparently "altruistic" behavior at the system level, which can prolong the survival of societies, could in fact include morally reprehensible forms of behavior and violence at the level of individual interactions. Rather than solely relying on cost-benefit analyses for modeling violence in human societies, we encourage deeper engagement with scholarship in the social sciences and humanities that highlights the complexity of modeling and representing even apparently simple human interactions, by revealing how deeply they are shaped by social, political, historical and institutional forces, both implicitly and explicitly $[1,2]$. In absence of such synthetic approaches, utilitarian perspectives on altruism and inter-generational benefits that undergird Hamilton's model of inclusive fitness for kin selection may be fundamentally reductionist and problematic as a paradigm for modeling human societies.

\section{References}

1. Ahmed, S.: Queer phenomenology: Orientations, objects, others. Duke University Press (2006)

2. Banerjee, P., Connell, R.: Gender theory as southern theory. In: Handbook of the Sociology of Gender, pp. 57-68. Springer (2018)

3. Carlin, N.F., Hölldobler, B.: The kin recognition system of carpenter ants (camponotus spp.). Behavioral Ecology and Sociobiology 19(2), 123-134 (1986)

4. Cole, B.J.: Dominance hierarchies in leptothorax ants. Science 212(4490), 83-84 (1981)

5. Dussutour, A., Simpson, S.J.: Communal nutrition in ants. Current Biology 19(9), 740-744 (2009)

6. Fisher, B., Ward, P.: Antweb. California Academy of Sciences (2002)

7. Franks, N.R., Richardson, T.: Teaching in tandem-running ants. Nature 439(7073), 153 (2006)

8. Grimm, V., Berger, U., Bastiansen, F., Eliassen, S., Ginot, V., Giske, J., GossCustard, J., Grand, T., Heinz, S.K., Huse, G., et al.: A standard protocol for describing individual-based and agent-based models. Ecological modelling 198(12), 115-126 (2006)

9. Hamilton, W.D.: The genetical evolution of social behaviour. i. Journal of theoretical biology 7(1), 1-16 (1964)

10. Hamilton, W.D.: The genetical evolution of social behaviour. ii. Journal of theoretical biology $\mathbf{7}(1), 17-52$ (1964)

11. Hamilton, W.D.: Altruism and related phenomena, mainly in social insects. Annual Review of Ecology and systematics 3(1), 193-232 (1972)

12. Holland, J.H.: Studying complex adaptive systems. Journal of Systems Science and Complexity 19(1), 1-8 (2006)

13. Holland, J.H.: Hidden orderhow adaptation builds complexity. No. 003.7 H6 (1995)

14. Hölldobler, B., Wilson, E.O.: The ants. Harvard University Press (1990) 
15. Hölldobler, B., Wilson, E.O.: The superorganism: the beauty, elegance, and strangeness of insect societies. WW Norton \& Company (2009)

16. Hölldobler, B., Wilson, E.O.: The leafcutter ants: civilization by instinct. WW Norton \& Company (2010)

17. Ihrig, S.: Justifying Genocide: Germany and the Armenians from Bismark to Hitler. Harvard University Press (2016)

18. Jackson, D.E., Ratnieks, F.L.: Communication in ants. Current biology 16(15), R570-R574 (2006)

19. Lahav, S., Soroker, V., Hefetz, A., Vander Meer, R.K.: Direct behavioral evidence for hydrocarbons as ant recognition discriminators. Naturwissenschaften 86(5), 246-249 (1999)

20. de Lazari-Radek, K., Singer, P.: The point of view of the universe: Sidgwick and contemporary ethics. OUP Oxford (2014)

21. Lenoir, A., Depickère, S., Devers, S., Christidès, J.P., Detrain, C.: Hydrocarbons in the ant lasius niger: from the cuticle to the nest and home range marking. Journal of chemical ecology 35(8), 913-921 (2009)

22. Lenoir, A., Fresneau, D., Errard, C., Hefetz, A.: Individuality and colonial identity in ants: the emergence of the social representation concept. In: Information processing in social insects, pp. 219-237. Springer (1999)

23. Liang, D., Silverman, J.: "you are what you eat": diet modifies cuticular hydrocarbons and nestmate recognition in the argentine ant, linepithema humile. Naturwissenschaften 87(9), 412-416 (2000)

24. Martin, K.: Umwelten ant model. CoMSES Computational Model Library, Evanston, https://www.comses.net/codebases/4499/releases/1.0.0/ (2015)

25. Martin, S.J., Vitikainen, E., Helanterä, H., Drijfhout, F.P.: Chemical basis of nestmate discrimination in the ant formica exsecta. Proceedings of the Royal Society of London B: Biological Sciences 275(1640), 1271-1278 (2008)

26. Moffett, M.W.: Ants \& the art of war. Scientific American 305(6), 84-89 (2011)

27. Moffett, M.W.: Supercolonies of billions in an invasive ant: what is a society? Behavioral Ecology 23(5), 925-933 (2012)

28. Page Jr, R.E., Metcalf, R.A.: Multiple mating, sperm utilization, and social evolution. The American Naturalist 119(2), 263-281 (1982)

29. Paré, D., Shanahan, M.C., Sengupta, P.: Queering complexity using multi-agent simulations. Proceedings of the 14th International Conference of Learning Sciences (ICLS 2020) TBD, TBD (2020)

30. Queller, D.C., Strassmann, J.E.: Kin selection and social insects. Bioscience 48(3), 165-175 (1998)

31. Seeley, T.D.: The honey bee colony as a superorganism. American Scientist 77(6), 546-553 (1989)

32. Sengupta, P., Wilensky, U.: Learning electricity with niels: Thinking with electrons and thinking in levels. International Journal of Computers for Mathematical Learning 14(1), 21-50 (2009)

33. Solvit, S.: Dimensions of war: Understanding war as a complex adaptive system. Editions L'Harmattan (2012)

34. Srinivasan, A.: Stop the robot apocalypse: the new utilitarians. London Review of Books 37(18), 3-6 (2015)

35. Strassmann, J.E., Queller, D.C.: Insect societies as divided organisms: the complexities of purpose and cross-purpose. Proceedings of the national academy of sciences 104(suppl 1), 8619-8626 (2007)

36. Tang, T., Ye, H.: The blessing of sexuality: evolution of altruism with mating preference. Journal of Artificial Societies and Social Simulation 19(2) (2016) 
37. Townsend, C., Begon, M., Harper, J., et al.: Essentials of ecology. No. Ed. 3, Blackwell Publishing (2008)

38. Trivers, R.: Social evolution. No. 156 T7 (1985)

39. Wagner, D., Tissot, M., Cuevas, W., Gordon, D.M.: Harvester ants utilize cuticular hydrocarbons in nestmate recognition. Journal of Chemical Ecology 26(10), 2245$2257(2000)$

40. Wheeler, W.M.: The ant-colony as an organism. Journal of Morphology 22(2), 307-325 (1911)

41. Whitehouse, M.E., Jaffe, K.: Ant wars: combat strategies, territory and nest defence in the leaf-cutting antatta laevigata. Animal Behaviour 51(6), 1207-1217 (1996)

42. Wilensky, U.: Netlogo (and netlogo user manual). Center for connected learning and computer-based modeling, Northwestern University. http://ccl. northwestern. edu/netlogo (1999)

43. Wilensky, U., Resnick, M.: Thinking in levels: A dynamic systems approach to making sense of the world. Journal of Science Education and technology 8(1), 3-19 (1999)

44. Wilson, E.O.: Sociobiology: The new synthesis. Belknap Press of Harvard U Press (1975)

45. Wilson, E.O., et al.: The insect societies. The insect societies. (1971)

46. Wright, M., Sengupta, P.: Modeling oligarchs' campaign donations and ideological preferences with simulated agent-based spatial elections. Journal of Artificial Societies and Social Simulation 18(2), 3 (2015)

47. Ydenberg, R.C., Giraldeau, L.A., Falls, J.B.: Neighbours, strangers, and the asymmetric war of attrition. Animal Behaviour 36(2), 343-347 (1988)

48. van Zweden, J.S., d Ettorre, P.: Nestmate recognition in social insects and the role of hydrocarbons. Insect hydrocarbons: biology, biochemistry and chemical ecology 11, 222-243 (2010) 